\title{
Fitted Mesh Method for Singularly Perturbed Robin Type Boundary Value Problem with Discontinuous Source Term
}

\author{
M. Chandru • V. Shanthi
}

Published online: 8 February 2015

(C) Springer India Pvt. Ltd. 2015

\begin{abstract}
In this paper, second order singularly perturbed convection-diffusion Robin type problem with a discontinuous source term is considered. Due to the discontinuity interior layers appears in the solution. A numerical method is constructed for this problem which involves an appropriate piecewise - uniform mesh for the boundary and interior layers. The method is shown to be parameter uniformly convergent with respect to the singular perturbation parameter. Numerical examples are presented to illustrate the theoretical results.
\end{abstract}

Keywords Singularly perturbed problem - Robin boundary condition ·

Discontinuous source term · Boundary layer · Interior layer .

Finite difference scheme

Mathematics Subject Classification $\quad 65$ L10 CR G1.7

\section{Introduction}

Singular perturbation problems (SPPs) model convection-diffusion process in applied mathematics that arise in diverse areas, including linearized Navier-Stokes equation at high Reynolds number and the drift-diffusion equation of semiconductor device modeling, heat and mass transfer at high Péclet number etc [1,2]. The novel aspect of the problem under consideration is that we take a source term in the differential equation which has a jump discontinuity at one or more points in the interior of the domain. This gives rise to an interior layer in the exact solution of the problem, in addition to the boundary layer at the outflow boundary point. Our goal is to construct an $\varepsilon$ uniform numerical method for solving this

M. Chandru $(\varangle) \cdot$ V. Shanthi

Department of Mathematics, National Institute of Technology,

Tiruchchirappalli 620 015, Tamil Nadu, India

e-mail: leochandru@gmail.com

V. Shanthi

e-mail: vshanthi@nitt.edu 
problem, that is a numerical method which generates $\varepsilon$ uniformly convergent numerical approximations to the solution and its derivatives. Note that problems with discontinuous data were treated theoretically, in the case of the solution of the convection diffusion with Dirichlet case problem $[3,4]$. In [5-8] the authors discussed a self-adjoint Dirichlet type problem with discontinuous source term. Shanthi et al. has examined two parameter singularly perturbed BVPs for second order ODEs with discontinuous source term in [9-11]. Singularly perturbed delay differential equation is examined in [12] on an adaptively generated grid.

Motivated by the works of [3-6] we, in the present paper, develop a computational method to solve SPBVPs for second order equations of the type:

$$
\begin{aligned}
L y(x) & \equiv \varepsilon y^{\prime \prime}(x)+a(x) y^{\prime}(x)-b(x) y(x)=f(x), \quad x \in\left(\Omega^{-} \cup \Omega^{+}\right), \\
B C_{1} y(0) & =\alpha_{1} y(0)-\beta_{1} \varepsilon y^{\prime}(0)=p, \quad B C_{2} y(1)=\alpha_{2} y(1)+\beta_{2} y^{\prime}(1)=q,
\end{aligned}
$$

where $\alpha_{1}, \beta_{1} \geq 0, \alpha_{1}+\beta_{1}>0, \beta_{2} \geq 0, \alpha_{2}>0$ and $\varepsilon>0$ is a small parameter. $a(x), b(x)$ are smooth functions $\bar{\Omega}$ such that

$$
\begin{aligned}
& a(x) \geq \alpha>0, \\
& b(x) \geq \beta \geq 0 .
\end{aligned}
$$

It is convenient to introduce the notation $\Omega=(0,1), \Omega^{-}=(0, d), \Omega^{+}=(d, 1), \quad d \in \Omega$ and to denote the jump at $d$ in any function with $[w](d)=w(d+)-w(d-)$. Further it is assumed that $f$ is sufficiently smooth on $\bar{\Omega} \backslash\{d\}$; a single discontinuity in the source term $f(x)$ occur at a point $d \in \Omega ; f(x)$ and its derivatives have jump discontinuity at the same point. In general this discontinuity gives rise to an interior layer in the second derivative of the exact solution of the problem. Because $f$ is discontinuous at $d$ the solution $y$ of (1)-(2) does not necessarily have a continuous second derivative at the point $d$. Thus $y \notin \mathcal{C}^{2}(\Omega)$. But the first derivative of the solution exists and is continuous. Boundary value problem of the type (1)-(2) model confinement of a plasma column by reaction pressure and geophysical fluid dynamics [13].

The nature of problem discussed in the Dirichlet case $\alpha_{1}=1, \beta_{1}=0, \alpha_{2}=1$ and $\beta_{2}=0$ and in the Neumann case $\alpha_{1}=0, \beta_{1}=1, \alpha_{2}=0$ and $\beta_{2}=1$ in [14]. For detailed study one may refer $[14,15]$.

Various methods are available in literature to obtain numerical solution to singularly perturbed differential equation (1) subject to Robin boundary conditions when $f$ is smooth on $\Omega$ [14,16-20]. Some recent works have been done in similar type of problem with smooth data as follows. The numerical integration method for general singularly perturbed boundary value problem with mixed boundary condition is presented in [21]. In [22] the authors have shown the advantages of Differential Quadrature Method (DQM) for finding the numerical solution [23]. Pratibhamoy Das et al. discussed on system of reaction diffusion differential equations for Robin or mixed type boundary value problems by a cubic spline approximation [24]. From this investigation the author considered a non-self adjoint Robin type problem with discontinuous source term, and obtained a parameter uniform convergent solution for equation (1)-(2).

"Some Analytical Results" section presents analytic behavior of the solution of the SPP (1)-(2). The present method is described in "Discrete Problem" section. "Error Analysis" section provides error estimates for the numerical solutions. Numerical examples are given in "Numerical Results" section. The paper ends with a discussion.

Throughout this paper, $C$ denotes a generic positive constant that is independent of nodal point $(i)$, mesh size $(h)$ and the singular perturbation parameter $(\varepsilon)$. We measure all functions 
in the maximum point wise norm, which we denote by $\|w\|_{D}=\sup _{x \in D}|w(x)|$, where $D$ is an open connected set.

\section{Some Analytical Results}

In this section, we provide a comparison principle for the following problem. Consequence of this principle gives the stability result for the same problem. By a suitable choice of the barrier function and the procedure adapted from $[3,16,25]$, we can prove the following theorems.

Theorem 1 The problem (1)-(2) has a solution $u \in \mathcal{C}^{1}(\bar{\Omega}) \cap C^{2}\left(\Omega^{-} \cup \Omega^{+}\right)$.

Theorem 2 (Comparison Principle) Suppose that a function $u \in \mathcal{C}^{1}(\bar{\Omega}) \cap \mathcal{C}^{2}\left(\Omega^{-} \cup \Omega^{+}\right)$, satisfies $B C_{1} u(0) \geq 0, B C_{2} u(1) \geq 0$ and $L u(x) \leq 0, \forall x \in \Omega^{-} \cup \Omega^{+}$and $\left[u^{\prime}\right](d) \leq 0$, then $u(x) \geq 0, \forall x \in \bar{\Omega}$.

An immediate consequence of the comparison principle is the following stability result.

Theorem 3 (Stability Result) Consider the BVP (1)-(2) subject to the conditions (3)-(4). If $u \in \mathcal{C}^{1}(\bar{\Omega}) \cap \mathcal{C}^{2}\left(\Omega^{-} \cup \Omega^{+}\right)$then

$$
\|u\|_{\bar{\Omega}} \leq C \max \left\{\left|B C_{1} u(0)\right|,\left|B C_{2} u(1)\right|,|L u|_{\Omega^{-} \cup \Omega^{+}}\right\} .
$$

Theorem 4 For each integer $k$, satisfying $0 \leq k \leq 4$, the solution $u$ of (1)-(2) satisfy the bounds

$$
\left\|u^{k}\right\|_{\bar{\Omega} \backslash\{d\}} \leq C \varepsilon^{-k}
$$

To establish the parameter-robust properties of the numerical methods involved in this paper, the following decomposition of $u$ into smooth $v$ and singular $w$ components is required. The smooth component $v$ is defined as the solution of

$$
\begin{aligned}
& a v_{0}^{\prime}-b v_{0}=f, \quad x \in \Omega^{-} \cup \Omega^{+}, \quad B C_{2} v_{0}(1)=q . \\
& a v_{1}^{\prime}-b v_{1}=-v_{0}^{\prime \prime}, \quad x \in \Omega^{-} \cup \Omega^{+}, \quad B C_{2} v_{1}(1)=0 .
\end{aligned}
$$

Note that $v=v_{0}+\varepsilon v_{1}+\varepsilon^{2} v_{2}$, where $v_{2} \in \mathcal{C}^{1}(\bar{\Omega}) \cap C^{2}\left(\Omega^{-} \cup \Omega^{+}\right)$and

$$
L v_{2}=-v_{1}^{\prime \prime}, \quad B C_{1} v_{2}(0)=v_{2}(d)=B C_{2} v_{2}(1)=0 .
$$

As in $[14,16]$, we can obtain the following bounds on the derivatives of $v$ for $k=1,2,3$.

$$
\|v\| \leq C,\left\|v^{(k)}\right\|_{\Omega^{-} \cup \Omega^{+}} \leq C\left(1+\varepsilon^{(2-k)}\right) .
$$

Note also that $\left|\left[v^{\prime}\right](d)\right| \leq C,\left|\left[v^{\prime \prime}\right](d)\right| \leq C$. Define the singular component of the decomposition as follows. Find $w \in \mathcal{C}^{0}(\Omega)$ such that

$$
\begin{gathered}
L w=0, \quad x \in \Omega^{-} \cup \Omega^{+}, \\
B C_{1} w(0)=y(0)-v(0), \quad B C_{2} w(1)=0, \quad\left[w^{\prime}\right](d)=-\left[v^{\prime}\right](d) .
\end{gathered}
$$


We can further decompose $w$ as

$$
w=w_{1}+w_{2},
$$

where $w_{1}$ lois the boundary layer function satisfying

$$
\begin{gathered}
w_{1} \in C^{2}(\Omega), \quad L w_{1}=0, x \in \Omega, \\
B C_{1} w(0)=y(0)-v(0), \quad B C_{2} w(1)=0
\end{gathered}
$$

and $w_{2}$ is the interior layer function satisfying

$$
\begin{gathered}
w_{2} \in C^{0}(\Omega), L w_{2}=0, \quad x \in \Omega^{-} \cup \Omega^{+}, \\
B C_{1} w_{2}(0)=0, \quad B C_{2} w_{2}(1)=0, \\
{\left[w_{2}^{\prime}\right](d)=-\left[v^{\prime}\right](d) .}
\end{gathered}
$$

The procedure adopted from [16] and suitable barrier function, one can obtain the results of the following Lemma 1, 2 and 3.

Lemma 1 [14,16] For each integer $k$ satisfying $0 \leq k \leq 3$, the solution $w_{1}$ of (8),(9) satisfies the bounds

$$
\left|w_{1}^{(k)}(x)\right| \leq C \varepsilon^{-k} e^{-\alpha x / \varepsilon}, \quad x \in \Omega^{-}
$$

where $C$ is a constant independent of $\varepsilon$.

Lemma 2 For each integer $k$, satisfying $1 \leq k \leq 3$, the solution $w_{2}$ of (10)-(12) satisfies the bounds

$$
\begin{gathered}
\left|w_{2}(x)\right| \leq C \\
\left|w_{2}^{k}(x)\right| \leq \begin{cases}C\left(\varepsilon^{(1-k)} e^{-\alpha x / \varepsilon}\right), & x \in \Omega^{-} \\
C\left(\varepsilon^{(1-k)} e^{-\alpha(x-d) / \varepsilon}\right), & x \in \Omega^{+},\end{cases}
\end{gathered}
$$

where $C$ is a constant independent of $\varepsilon$.

\section{Discrete Problem}

A fitted mesh method for problem (1)-(2) is now described. On $\Omega$ a piecewise uniform mesh of $\mathrm{N}$ mesh intervals is constructed as follows. The interval $\Omega$ is subdivided into four subintervals.

$$
\left[0, \tau_{1}\right] \cup\left[\tau_{1}, d\right] \cup\left[d, d+\tau_{2}\right] \cup\left[d+\tau_{2}, 1\right]
$$

for some $\tau_{1}, \tau_{2}$ that satisfies $0<\tau_{1} \leq d / 2,0 \leq \tau_{2} \leq \frac{1-d}{2}$. On each subinterval a uniform mesh with $\frac{N}{4}$ mesh-intervals is placed. The interior points of the mesh are denoted by

$$
\Omega_{\varepsilon}^{N}=\left\{x_{i}: 1 \leq i \leq \frac{N}{2}-1\right\} \cup\left\{x_{i}: \frac{N}{2}+1 \leq i \leq N-1\right\} .
$$

Clearly $x_{N / 2}=d$ and $\bar{\Omega}_{\varepsilon}^{N}=\left\{x_{i}\right\}_{0}^{N}$. Note that this mesh is a uniform mesh when $\tau_{1}=\frac{d}{2}$ and $\tau_{2}=\frac{1-d}{2}$. It is fitted to the singular perturbation problem (13)-(14) by choosing $\tau_{1}$ and $\tau_{2}$ to be the following functions of $\mathrm{N}$ and $\varepsilon$ 


$$
\tau_{1}=\min \left\{\frac{d}{2},(\varepsilon / \alpha) \ln N\right\} \quad \text { and } \quad \tau_{2}=\min \left\{\frac{1-d}{2},(\varepsilon / \alpha) \ln N\right\} .
$$

We introduce the following notation for four mesh widths

$$
h_{1}=4 \tau_{1} / N, \quad H_{1}=4\left(d-\tau_{1}\right) / N, \quad h_{2}=4 \tau_{2} / N, \quad H_{2}=4\left(1-d-\tau_{2}\right) / N .
$$

On the piecewise-uniform mesh $\bar{\Omega}_{\varepsilon}^{N}$ a standard centered finite difference operator is used. Then the fitted mesh method for (1)-(2) is

$$
\begin{gathered}
L_{i}^{y} \equiv \varepsilon \delta^{2} y_{i}+a\left(x_{i}\right) D^{+} y_{i}-b\left(x_{i}\right) y_{i}=f\left(x_{i}\right), \quad \forall x_{i} \in \Omega_{\varepsilon}^{N} \\
B C_{1}^{N} y_{0}=\alpha_{1} y_{0}-\beta_{1} \varepsilon D^{+} y_{0}=r, \quad B C_{2}^{N} y_{N}=\alpha_{2} y_{N}+\beta_{2} D^{-} y_{N}=s, \\
D^{-} y_{\varepsilon}\left(x_{N / 2}\right)=D^{+} y_{\varepsilon}\left(x_{N / 2}\right),
\end{gathered}
$$

where,

$$
\begin{aligned}
\delta^{2} Z_{i} & =\left(\frac{Z_{i+1}-Z_{i}}{x_{i+1}-x_{i}}-\frac{Z_{i}-Z_{i-1}}{x_{i}-x_{i-1}}\right) \frac{2}{x_{i+1}-x_{i-1}}, \\
D^{+} Z_{i} & =\frac{Z_{i+1}-Z_{i}}{x_{i+1}-x_{i}}, \text { and } D^{-} Z_{i}=\frac{Z_{i}-Z_{i-1}}{x_{i}-x_{i-1}} .
\end{aligned}
$$

Lemma 3 Suppose that a mesh function $Z$ satisfies $B C_{1}^{N} Z\left(x_{0}\right) \geq 0, B C_{2}^{N} Z\left(x_{N}\right) \geq$ $0, L^{N} Z\left(x_{i}\right) \geq 0$ for all $x_{i} \in \Omega_{\varepsilon}^{N}$, and $D^{+} Z\left(x_{N / 2}\right)-D^{-} Z\left(x_{N / 2}\right) \leq 0$, then $Z\left(x_{i}\right) \geq 0$ for all $x_{i} \in \bar{\Omega}_{\varepsilon}^{N}$.

\section{Error Analysis}

Define the function $V$ to be the solution of

$$
\begin{gathered}
L^{N} V=f\left(x_{i}\right), \quad \forall x_{i} \in \Omega_{\varepsilon}^{N} \\
B C_{1}^{N} V(0)=B C_{1} v(0), \quad V(d)=v(d), \quad B C_{2}^{N} V(1)=B C_{2} v(1) .
\end{gathered}
$$

Using a standard stability and consistency argument coupled with the obvious barrier functions on each interval $[0, d],[d, 1]$ separately, by [14], one can deduce that

$$
\left|V\left(x_{i}\right)-v\left(x_{i}\right)\right| \leq \begin{cases}C N^{-1}\left(d-x_{i}\right), & x_{i} \leq d, \\ C N^{-1}\left(1-x_{i}\right), & x_{i} \geq d .\end{cases}
$$

We define $W$ to be the solution of

$$
\begin{gathered}
L^{N} W=0, \quad \forall x_{i} \in \Omega_{\varepsilon}^{N} \\
B C_{1}^{N} W(0)=B C_{1} w(0), \quad B C_{2}^{N} W(1)=B C_{2} w(1), \quad[D W(d)]=-[D V(d)],
\end{gathered}
$$

where throughout this section, we denote the jump in the discrete derivative of mesh function $Z$ at the point $x_{i}=d$ by

$$
[D Z(d)]=D^{+} Z(d)-D^{-} Z(d) .
$$

Analogously to the continuous case we can further decompose $W$ as

$$
W=W_{1}+W_{2}
$$


where $W_{1}$ ( the discrete analogue of the boundary layer function $w_{1}$ ) is defined as solution of

$$
\begin{aligned}
L^{N} W_{1} & =0, \quad \forall x_{i} \in \Omega_{\varepsilon}^{N} \cup\{d\} \\
B C_{1}^{N} W_{1}(0) & =B C_{1} w_{1}(0), \quad B C_{2}^{N} W_{1}(1)=0
\end{aligned}
$$

and $W_{2}$ ( the discrete analogue of the interior layer function $w_{2}$ ) is defined as solution of

$$
\begin{aligned}
L^{N} W_{2} & =0, \quad \forall x_{i} \in \Omega_{\varepsilon}^{N} \\
B C_{1}^{N} W_{2}(1) & =0, \quad B C_{2}^{N} W_{2}(1)=0 \\
{\left[D W_{2}(d)\right] } & =-[D V(d)]-\left[D W_{1}(d)\right]
\end{aligned}
$$

As in [14],

$$
\left|W_{1}-w_{1}\right| \leq C N^{-1} \ln N, \quad x_{i} \in \Omega_{\varepsilon}^{N} \cup\{d\} .
$$

And $\left|W_{1}\right| \leq C N^{-1}$ for all $x_{i} \geq d$. Note that the jump at $x=d$ in the derivative of weak interior layer function $w_{2}$ is bounded. The next lemma establishes the discrete counterpart of this.

The procedure given in page. 140 of [3] is also valid for $B C_{1}^{N} y_{0}$ and $B C_{2}^{N} y_{N}$ with the same barrier function. Therefore, from Lemma 4 and Lemma 5 of [3], we obtain

Lemma 4 Assuming $N \geq 8$ then

$$
\left|W_{2}\left(x_{i}\right)-w_{2}\left(x_{i}\right)\right| \leq C\left(\varepsilon+N^{-1}\right) \leq C N^{-1} \ln N .
$$

Let us first consider the magnitude of the truncation error at the point of discontinuity $x=d$. Using the procedure following in [3] and the derivative estimate established in [14] the following result can be obtain

$$
|[D(V-v)(d)]| \leq C(\varepsilon N)^{-1} \quad \text { and } \quad\left|\left[D\left(W_{2}-w_{2}\right)(d)\right]\right| \leq C N^{-1} \ln N / \varepsilon .
$$

Define the barrier function $\phi_{i}[3]$ to be

$$
\phi_{i}=C N^{-1} \ln N\left\{\begin{array}{ll}
1, & x_{i} \leq d \\
\psi, & x_{i} \geq d
\end{array}+C N^{-1} \ln N\left(1-x_{i}\right),\right.
$$

where $\psi$ is the solution of

$$
\begin{gathered}
\varepsilon \delta^{2} \psi+\alpha D^{+} \psi-b \psi=0, \quad x_{i} \in \Omega^{+} \cap \Omega_{\varepsilon}^{N} \\
\psi(d)=1, \quad B C_{2}^{N} \psi(1)=0 .
\end{gathered}
$$

Theorem 5 Let $U, u$ be the solution of $L^{N}, L$ respectively. Assume that $N \geq 8$, then

$$
\begin{aligned}
\|\tilde{U}-u\|_{\bar{\Omega}} & \leq C N^{-1} \ln N, \\
\varepsilon\left\|D^{+} \tilde{U}-u^{\prime}\right\|_{\bar{\Omega}} & \leq C N^{-1} \ln N,
\end{aligned}
$$

where $\tilde{U}$ is the piecewise linear interpolant of $U$ on $\bar{\Omega}$ and $C$ is a constant independent of $N$ and $\varepsilon$.

Proof Using (15), (21) and Lemma 4 it is easy to derive 
Table 1 Maximum pointwise error $E_{\varepsilon}^{N}$ and order of convergence $\rho^{N}$ for the Example 1

\begin{tabular}{|c|c|c|c|c|c|c|}
\hline \multirow[t]{2}{*}{$\varepsilon$} & \multicolumn{6}{|c|}{ Number of mesh points } \\
\hline & $2^{6}$ & $2^{7}$ & $2^{8}$ & $2^{9}$ & $2^{10}$ & $2^{11}$ \\
\hline $2^{0}$ & $1.1329 \mathrm{E}-02$ & $5.5712 \mathrm{E}-03$ & $2.6949 \mathrm{E}-03$ & $1.2574 \mathrm{E}-03$ & $5.3885 \mathrm{E}-04$ & $1.7961 \mathrm{E}-04$ \\
\hline $2^{-1}$ & $6.8319 \mathrm{E}-03$ & $3.2397 \mathrm{E}-03$ & $1.5367 \mathrm{E}-03$ & $7.0975 \mathrm{E}-04$ & $3.0258 \mathrm{E}-04$ & $1.0059 \mathrm{E}-04$ \\
\hline $2^{-2}$ & $8.7403 E-03$ & $4.4761 \mathrm{E}-03$ & $2.2085 \mathrm{E}-03$ & $1.0406 \mathrm{E}-03$ & $4.4812 \mathrm{E}-04$ & $1.4973 \mathrm{E}-04$ \\
\hline $2^{-3}$ & $2.1626 \mathrm{E}-02$ & $1.0694 \mathrm{E}-02$ & $5.1847 \mathrm{E}-03$ & $2.4216 \mathrm{E}-03$ & $1.0382 \mathrm{E}-03$ & $3.4615 \mathrm{E}-04$ \\
\hline $2^{-4}$ & $2.0405 \mathrm{E}-02$ & $1.0005 \mathrm{E}-02$ & $4.8332 \mathrm{E}-03$ & $2.2538 \mathrm{E}-03$ & $9.6554 \mathrm{E}-04$ & $3.2179 \mathrm{E}-04$ \\
\hline $2^{-5}$ & $1.7981 \mathrm{E}-02$ & $1.0249 \mathrm{E}-02$ & $5.6613 \mathrm{E}-03$ & $2.2538 \mathrm{E}-03$ & $1.4016 \mathrm{E}-03$ & $5.1662 \mathrm{E}-04$ \\
\hline $2^{-6}$ & $2.0072 \mathrm{E}-02$ & $1.1136 \mathrm{E}-02$ & $6.0306 \mathrm{E}-03$ & $3.1191 \mathrm{E}-03$ & $1.4580 \mathrm{E}-03$ & $5.2160 \mathrm{E}-04$ \\
\hline $2^{-7}$ & $2.2061 \mathrm{E}-02$ & $1.2045 \mathrm{E}-02$ & $6.4104 \mathrm{E}-03$ & $3.2729 \mathrm{E}-03$ & $1.5180 \mathrm{E}-03$ & $5.4072 \mathrm{E}-04$ \\
\hline $2^{-8}$ & $2.3563 \mathrm{E}-02$ & $1.2830 \mathrm{E}-02$ & $6.7717 \mathrm{E}-03$ & $3.4147 \mathrm{E}-03$ & $1.5689 \mathrm{E}-03$ & $5.5587 \mathrm{E}-04$ \\
\hline $2^{-9}$ & $2.4610 \mathrm{E}-02$ & $1.3410 \mathrm{E}-02$ & $7.0775 \mathrm{E}-03$ & $3.5532 \mathrm{E}-03$ & $1.6202 \mathrm{E}-03$ & $5.6995 \mathrm{E}-04$ \\
\hline $2^{-10}$ & $2.5198 \mathrm{E}-02$ & $1.3801 \mathrm{E}-02$ & $7.3129 \mathrm{E}-03$ & $3.6779 \mathrm{E}-03$ & $1.6744 \mathrm{E}-03$ & $5.8585 \mathrm{E}-04$ \\
\hline $2^{-11}$ & $2.5506 \mathrm{E}-02$ & $1.4019 \mathrm{E}-02$ & $7.4585 \mathrm{E}-03$ & $3.7681 \mathrm{E}-03$ & $1.7215 \mathrm{E}-03$ & $6.0295 \mathrm{E}-04$ \\
\hline $2^{-12}$ & $2.5658 \mathrm{E}-02$ & $1.4128 \mathrm{E}-02$ & $7.5359 \mathrm{E}-03$ & $3.8225 \mathrm{E}-03$ & $1.7536 \mathrm{E}-03$ & $6.1686 \mathrm{E}-04$ \\
\hline$E^{N}$ & $2.5658 \mathrm{E}-02$ & $1.4128 \mathrm{E}-02$ & $7.5359 \mathrm{E}-03$ & $3.8225 \mathrm{E}-03$ & $1.7536 \mathrm{E}-03$ & $6.1686 \mathrm{E}-04$ \\
\hline$\rho$ & $8.6085 \mathrm{E}-01$ & $9.0671 \mathrm{E}-01$ & $9.7927 \mathrm{E}-01$ & $1.1242 \mathrm{E}+00$ & $1.5073 \mathrm{E}+00$ & \\
\hline
\end{tabular}

$$
\|\tilde{U}-u\|_{\bar{\Omega}} \leq C N^{-1} \ln N
$$

To extend this one can follow the procedure adopted in [14] and [15]. To obtain the bound on the errors in approximations to the scaled derivative, we apply the arguments from [15] and the procedure adopted in [3] separately on each subdomain $[0, d]$ and $[d, 1]$ to get

$$
\begin{aligned}
\varepsilon\left\|\bar{D}^{+} y-y^{\prime}\right\|_{\bar{\Omega}} & \leq C N^{-1} \ln N \\
\varepsilon\left\|\bar{D}^{+} V-v^{\prime}\right\|_{\bar{\Omega}} & \leq C N^{-1} \ln N \\
\varepsilon\left\|\bar{D}^{+}\left(W_{1}-w_{1}\right)\right\|_{[0, d)} & \leq C N^{-1} \ln N
\end{aligned}
$$

and from the proof of Lemma 4 of [3]

$$
\varepsilon\left\|\bar{D}^{+}\left(W_{1}-w_{1}\right)\right\|_{[d, 1)} \leq C N^{-1} \ln N .
$$

On the subdomain $[d, 1]$, we also apply these arguments to get

$$
\varepsilon\left\|\bar{D}^{+}\left(W_{2}-w_{2}\right)\left(x_{i}\right)\right\| \leq C N^{-1} \ln N, \quad x_{i} \geq d .
$$

Based on the simple argument given in [3]. We can prove

$$
\varepsilon\left\|\bar{D}^{+} W_{2}-w_{2}\left(x_{i}\right)\right\| \leq C N^{-1} \ln N, x_{i}<d .
$$

which completes the proof. 
Table 2 Maximum pointwise error $E_{\varepsilon}^{N}$ and order of convergence $\rho^{N}$ for the Example 2

\begin{tabular}{|c|c|c|c|c|c|c|}
\hline \multirow[t]{2}{*}{$\varepsilon$} & \multicolumn{6}{|c|}{ Number of mesh points } \\
\hline & $2^{6}$ & $2^{7}$ & $2^{8}$ & $2^{9}$ & $2^{10}$ & $2^{11}$ \\
\hline $2^{0}$ & $6.6163 \mathrm{E}-02$ & $3.2658 \mathrm{E}-02$ & $1.5827 \mathrm{E}-02$ & $7.3916 \mathrm{E}-03$ & $3.1691 \mathrm{E}-03$ & $1.0566 \mathrm{E}-03$ \\
\hline $2^{-1}$ & $6.4196 \mathrm{E}-02$ & $3.1037 \mathrm{E}-02$ & $1.4876 \mathrm{E}-02$ & $6.9081 \mathrm{E}-03$ & $2.9532 \mathrm{E}-03$ & $9.8316 \mathrm{E}-04$ \\
\hline $2^{-2}$ & $2.1351 \mathrm{E}-01$ & $1.0537 \mathrm{E}-01$ & $5.1051 \mathrm{E}-02$ & $2.3838 \mathrm{E}-02$ & $1.0219 \mathrm{E}-02$ & $3.4070 \mathrm{E}-03$ \\
\hline $2^{-3}$ & $3.4614 \mathrm{E}-01$ & $1.6986 \mathrm{E}-01$ & $8.2076 \mathrm{E}-02$ & $3.8274 \mathrm{E}-02$ & $1.6397 \mathrm{E}-02$ & $5.4647 \mathrm{E}-03$ \\
\hline $2^{-4}$ & $4.7760 \mathrm{E}-01$ & $2.4152 \mathrm{E}-01$ & $1.1853 \mathrm{E}-01$ & $5.5717 \mathrm{E}-02$ & $2.3969 \mathrm{E}-02$ & $8.0047 \mathrm{E}-03$ \\
\hline $2^{-5}$ & $8.4233 \mathrm{E}-01$ & $4.5042 \mathrm{E}-01$ & $2.2634 \mathrm{E}-01$ & $1.0795 \mathrm{E}-01$ & $4.6763 \mathrm{E}-02$ & $1.5672 \mathrm{E}-02$ \\
\hline $2^{-6}$ & $9.0232 \mathrm{E}-01$ & $5.4579 \mathrm{E}-01$ & $3.0795 \mathrm{E}-01$ & $1.6300 \mathrm{E}-01$ & $7.7457 \mathrm{E}-02$ & $2.8493 \mathrm{E}-02$ \\
\hline $2^{-7}$ & $9.3284 \mathrm{E}-01$ & $5.6182 \mathrm{E}-01$ & $3.1678 \mathrm{E}-01$ & $1.6732 \mathrm{E}-01$ & $7.8777 \mathrm{E}-02$ & $2.8312 \mathrm{E}-02$ \\
\hline $2^{-8}$ & $9.4993 \mathrm{E}-01$ & $5.7117 \mathrm{E}-01$ & $3.2238 \mathrm{E}-01$ & $1.7024 \mathrm{E}-01$ & $8.0147 \mathrm{E}-02$ & $2.8806 \mathrm{E}-02$ \\
\hline $2^{-9}$ & $9.5888 \mathrm{E}-01$ & $5.7609 \mathrm{E}-01$ & $3.2530 \mathrm{E}-01$ & $1.7176 \mathrm{E}-01$ & $8.0859 \mathrm{E}-02$ & $2.9063 \mathrm{E}-02$ \\
\hline $2^{-10}$ & $9.6347 \mathrm{E}-01$ & $5.7862 \mathrm{E}-01$ & $3.2680 \mathrm{E}-01$ & $1.7253 \mathrm{E}-01$ & $8.1223 \mathrm{E}-02$ & $2.9195 \mathrm{E}-02$ \\
\hline $2^{-11}$ & $9.6580 \mathrm{E}-01$ & $5.7991 \mathrm{E}-01$ & $3.2756 \mathrm{E}-01$ & $1.7293 \mathrm{E}-01$ & $8.1407 \mathrm{E}-02$ & $2.9261 \mathrm{E}-02$ \\
\hline $2^{-12}$ & $9.6698 \mathrm{E}-01$ & $5.8056 \mathrm{E}-01$ & $3.2795 \mathrm{E}-01$ & $1.7313 \mathrm{E}-01$ & $8.1501 \mathrm{E}-02$ & $2.9295 \mathrm{E}-02$ \\
\hline$E^{N}$ & $9.6698 \mathrm{E}-01$ & $5.8056 \mathrm{E}-01$ & $3.2795 \mathrm{E}-01$ & $1.7313 \mathrm{E}-01$ & $8.1501 \mathrm{E}-02$ & $2.9295 \mathrm{E}-02$ \\
\hline$\rho$ & $7.3604 \mathrm{E}-01$ & $8.2399 \mathrm{E}-01$ & $9.2161 \mathrm{E}-01$ & $1.0870 \mathrm{E}+00$ & $1.4762 \mathrm{E}+00$ & \\
\hline
\end{tabular}

\section{Numerical Results}

In this section, two examples are given to illustrate the computational methods discussed in this paper.

Consider the singularly perturbed BVPs with discontinuous source term:

\section{Example 1}

$$
\begin{aligned}
\varepsilon y^{\prime \prime}(x)+y^{\prime}(x) & =f(x), \quad x \in \Omega^{-} \cup \Omega^{+}, \\
y(0)-\varepsilon y^{\prime}(0) & =1, \quad y(1)+y^{\prime}(1)=-1,
\end{aligned}
$$

where

$$
f(x)= \begin{cases}0.7, & \text { for } 0 \leq x \leq 0.5 \\ -0.6, & \text { for } 0.5<x \leq 1\end{cases}
$$

Example 2

$$
\begin{gathered}
\varepsilon y^{\prime \prime}(x)+\frac{1}{1+x} y^{\prime}(x)=f(x), \quad x \in \Omega^{-} \cup \Omega^{+}, \\
y(0)-\varepsilon y^{\prime}(0)=1, \quad y(1)+y^{\prime}(1)=1,
\end{gathered}
$$

where

$$
f(x)= \begin{cases}1+x, & \text { for } 0 \leq x \leq 0.5 \\ 4.0, & \text { for } 0.5<x \leq 1\end{cases}
$$

which validate the theoretical results established in the previous section. The nodal errors and orders of convergence are estimated using the interpolation principle. Define the nodal 


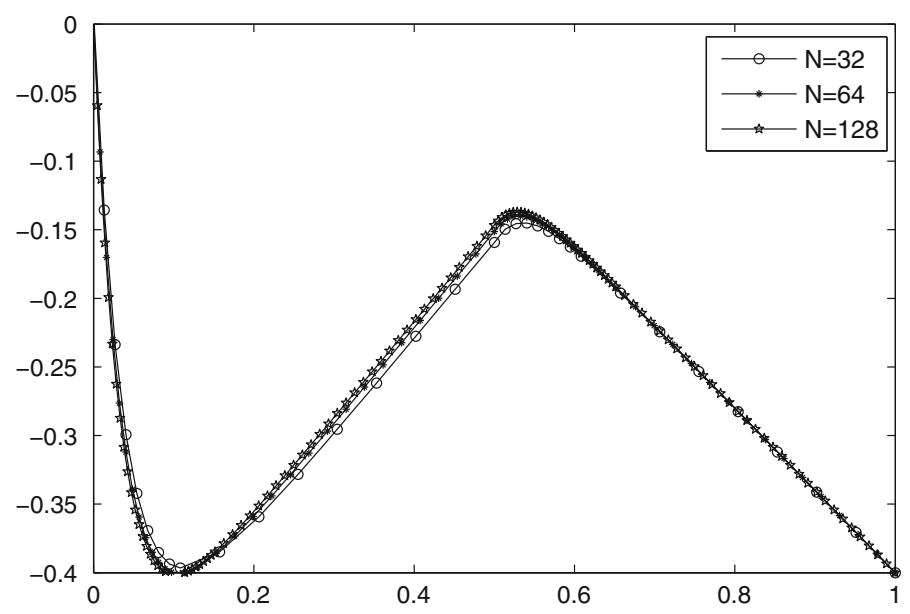

Fig. 1 Solution plot of $N=32,64,128$ and $\varepsilon=2^{-5}$ for the Problem 1

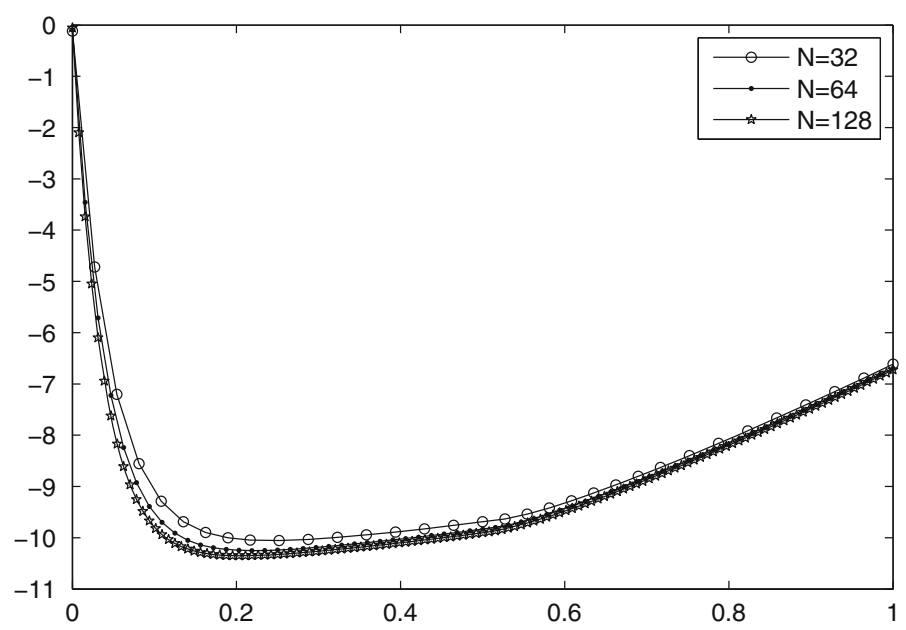

Fig. 2 Solution plot of $N=32,64,128$ and $\varepsilon=2^{-5}$ for the Problem 2

error of interpolation principle, the difference between the numerical solutions for various values of $N$ and $N=4096$.

$$
E_{\varepsilon}^{N} \equiv \max _{x_{i} \in \bar{\Omega}_{\varepsilon}^{N}}\left|U_{\varepsilon}^{N}\left(x_{i}\right)-\bar{U}_{\varepsilon}^{4096}\left(x_{i}\right)\right| \text { and } E^{N}=\max _{\varepsilon} E_{\varepsilon}^{N} .
$$

From these quantities the parameter-robust order of convergence are computed from

$$
\rho^{N}=\log _{2}\left(\frac{E^{N}}{E^{2 N}}\right) .
$$

Tables 1 and 2 present values of the maximum pointwise error $E_{\varepsilon}^{N}$ and order of convergence $\rho^{N}$ for the Examples 1 and 2 respectively. The numerical solutions of the Example 1 and 2 are presented in Figs. 1 and 2. 


\section{Conclusion}

A singularly perturbed second-order ODEs of Robin type BVPs with discontinuous source term is considered. Due to discontinuity in the source term there is an interior layer occurring. To fit the interior and boundary layer a suitable piecewise uniform mesh is constructed. The numerical method generates $\varepsilon$-uniform convergence in the global maximum norm of the approximations. The numerical approximation coincides with theoretical result.

Acknowledgments The authors wishes to acknowledge National Board of Higher Mathematics (NBHM), DAE, Mumbai, India for its financial support of project 2/48(5)2010-R\&D II/8896. We thank the unknown referees for their valuable comments, also we thank them for refering in a short span of time

\section{References}

1. Roos, H.-G., Stynes, M., Tobiska, L.: Numerical Methods for Singularly Perturbed Differential Equations. Springer, New York (1996)

2. Doolan, E.P., Miller, J.J.H., Schilders, W.H.A.: Uniform Numerical Methods for Problems with Initial and Boundary Layers. Boole Press, Dublin (1980)

3. Farrell, P.A., Hegarty, A.F., Miller, J.J.H., O'Riordan, E., Shishkin, G.I.: Singularly perturbed convection diffusion problems with boundary and weak interior layers. J. Comput. Appl. Math. 166(1), 133-151 (2004)

4. Farrell, P.A., Hegarty, A.F., Miller, J.J.H., O’Riordan, E., Shishkin, G.I.: Global maximum norm parameter-uniform numerical method for a singularly perturbed convection-diffusion problem with discontinuous convection coefficient. Math. Comput. Model. 40(11-12), 1375-1392 (2004)

5. Chandru, M., Shanthi, V.: A boundary value technique for singularly perturbed boundary value problem of reaction-diffusion with non-smooth data, Journal of Engineering Science and Technology, Special Issue on ICMTEA 2013 Conference, 32-45, (2014)

6. Chandru, M., Prabha, T., Shanthi, V.: A hybrid difference scheme for a second-order singularly perturbed reaction-diffusion problem with non-smooth data. Int. J. Appl. Comput. Math. (2014). doi:10.1007/ s40819-014-0004-8

7. Roos, H.G., Zarin, H.: A second order scheme for singularly perturbed differential equations with discontinuous source term. J. Numer. Math. 10(4), 275-289 (2010)

8. Farrell, P.A., Miller, J.J.H., O’Riordan, E., Shishkin, G.I.: Singularly perturbed differential equations with discontinuous source terms. In: Appeared in Proceedings of Workshop'98, Lozenetx, Bulgaria, Aug 27-31, 147-156 (1998)

9. Shanthi, V., Ramanujam, N., Natesan, S.: Fitted mesh method for singularly perturbed reactionconvection-diffusion problems with boundary and interior layers. J. Appl. Math. Comput. 22(1-2), 49-65 (2006)

10. Shanthi, V., Ramanujam, N.: Asymptotic numerical method for singularly perturbed fourth order ordinary differential equations of convection-diffusion type. J. Appl. Math. comput. 133, 559-579 (2002)

11. Shanthi, V., Ramanujam, N.: A Boundary value technique for boundary value problems for singularly perturbed fourth order ordinary differential equations. J. Comput. Math. appl. 47, 1673-1688 (2004)

12. Mohapatra, J., Natesan, S.: Uniform convergence analysis of finite difference scheme for singularly perturbed delay differential equation on an adaptively generated grid. Numer. Math. 3(1), 1-22 (2010)

13. Chin, R.C.Y., Krasny, R.: A hybrid asymptotic finite element method for stiff two-point boundary value problems. SIAM J. Sci. Stat. Comput. 4, 229-243 (1983)

14. Ansari, Ali R., Hegarty, Alan F.: Numerical solution of a convection diffusion problem with Robin boundary conditions. J. Comput. Appl. Math. 156(1), 221-238 (2003)

15. Farrell, P.A., Hegarty, A.F., Miller, J.J.H., O’ Riordan, E., Shishkin, G.I.: Robust Computational Techniques for Boundary Layers. Chapman and Hall/ CRC, Boca Raton (2000)

16. Natesan, S., Ramanujam, N.: Booster method for singularly perturbed Robin problems i. Int. J. Comput. Math. 76(2), 191-202 (2000)

17. Natesan, S., Bawa, R.K.: Second-order numerical scheme for singularly perturbed reaction-diffusion Robin problems. J. Numer. Anal. Ind. Appl. Math. 2(3-4), 177-192 (2007) 
18. Natesan, S., Ramanujam, N.: A Booster method for singular perturbation problems arising in chemical reactor theory. Appl. Math. Comput. 100(1), 27-48 (1999)

19. Natesan, S., Ramanujam, N.: 'Shooting method' for the solution of singularly perturbed two-point boundary value problems having less severe boundary layers. Appl. Math. Comput. 133(2-3), 623-641 (2002)

20. Natesan, S., Ramanujam, N.: An asymptotic numerical method for singularly perturbed Robin problems-I. Appl. Math. Comput. 126, 97-107 (2002)

21. Andargie, A., Reddy, Y.N.: Numerical integration method for singular perturbation problems with mixed boundary conditions. J. Appl. Math. Inform. 26(5-6), 1273-1287 (2008)

22. Prasad, H.S., Reddy, Y.N.: Numerical treatment of singularly perturbed two-point boundary value problems with mixed condition using differential quadrature method. Int. J. Appl. Sci. Eng. 9(3), 207-221 (2011)

23. Mohapatra, Jugal: Natesan, Srinivasan: Parameter-uniform numerical methods for singularly perturbed mixed boundary value problems using grid equidistribution. J. Appl. Math. Comput. 37, 247-265 (2011)

24. Das, Pratibhamoy: Natesan, Srinivasan: A uniformly convergent hybrid scheme for singularly perturbed system of reaction-diffusion Robin type boundary-value problems. J. Appl. Math. Comput. 41(1-2), 447-471 (2013)

25. Nayfeh, A.H.: Introduction to Perturbation Methods. Wiley, New York (1981) 